\title{
Assessment of the Diagnostic Performance of Normal and Irradiated Schistosoma mansoni Cercarial Antigens using ELISA
}

\author{
Mona Mohammed EL-Derbawy ${ }^{1}$, Eman Naser Hafez ${ }^{2 *}$, \\ Mona Abdel Daym Abd Rabbo ${ }^{1}$, Nagwa Ibrahim Toaleb ${ }^{3}$, \\ Saedia Abdel Hady Sayed El-Ahl ${ }^{1}$ and Bahaa Eldkeen Wade EL-Aswad ${ }^{4}$ \\ ${ }^{1}$ Parasitology Department, Faculty of Medicine for Girls, Al-Azhar University, Egypt \\ ${ }^{2}$ Health Radiation Research Department, National Center for Radiation, Research and \\ Technology, Egyptian Atomic Energy Authority, Cairo, Egypt \\ ${ }^{3}$ Veterinary Research Division, National Research Centre, Egypt \\ ${ }^{4}$ Parasitology department, Faculty of Medicine, Menoufiya University, Egypt
}

\begin{abstract}
A B S T RA C T
Irradiation of Schistosoma mansoni cercariae induces structural and motor changes in its surface molecules converting it from poor immunogenic to highly immunogenic surface. This study aims to comparatively analyze normal and gamma radiation-attenuated Schistosoma mansoni cercarial antigens (SCA) by ELISA for serodiagnosis. Total IgG ELISA was assessed to evaluate the potency of two parts of Schistosoma mansoni ceracrial antigens (2000 each) one normal and other exposed to attenuating dose of $400 \mathrm{~Gy}$ gamma radiations. The study was conducted on fifty two individual sera samples divided as follows: Group I included 21 obtained from patients who were positive for schistosomiasis and negative for other parasitic infections; Group II included 18 sera from patients who were negative for schistosomiasis and positive for other parasitic infections; (3) fascioliasis, (5) amoebiasis, (7) toxoplasmosis and (3) hydatidosis and Group III included (13) sera from healthy individuals all were diagnosed parasitologically and serologically. Although both antigens achieved $100 \%$ sensitivity and negative predictive value (NPP), irradiated SCA revealed better specificity, accuracy and positive predictive value (PPV) of $87 \%, 92.3 \%$ and $84 \%$, respectively. While normal SCA revealed $83.9 \%, 90.4 \%$ and $80.7 \%$, respectively. It was concluded that irradiated SCA achieved better remarkable ODs than normal SCA and better specificity due to low cross-reactivity with other parasites. S0 it can be used as a tool for diagnosis of chronic schistosomiasis.
\end{abstract}

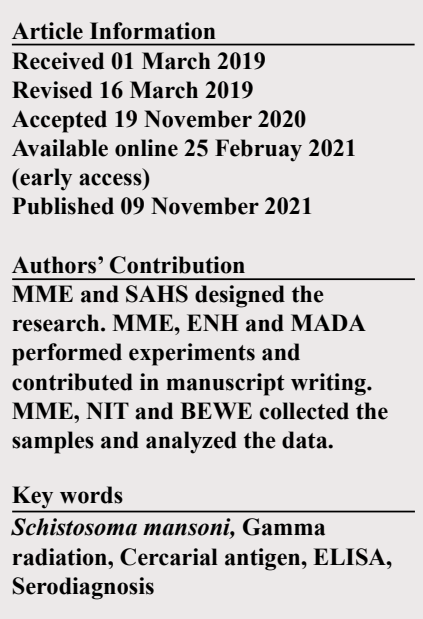

\section{INTRODUCTION}

$\mathrm{S}_{\mathrm{s}}$ chistosomiasis is a tropical disease where induction of protective immunity and elimination can be achieved by sustainable surveillance and implantation of a sensitive diagnostic method for control (Amin et al., 2017). Currently used diagnostics as Kato-Katz fecal smear lack sensitivity to accurately determine the prevalence of it in areas with low infection as it is affected by day and day variation in egg excretion (He et al., 2018).

One of advanced strategies to diagnose atypical forms of Schistosome is detection of antibodies which can be used as biomarker of progression of the disease (Ludolf et al., 2014). However, persistence of antibodies in the host makes these tests limited in detection of early infection and in assessing response to treatment so a need for more

\footnotetext{
* Corresponding author: eman_naser612@hotmail. com

0030-9923/2022/0001-0001 \$9.00/0

Copyright 2022 Zoological Society of Pakistan
}

sensitive assays is highly desirable (Siqueira et al., 2011; Cavalcanti et al., 2013). The association of antibody and antigen detection assays potentiates the diagnosis. Enzyme linked immunosorbent assay (ELISA), indirect haemagglutination, or immunofluorescence are the most current serological tests to detect antibodies against schistosomal antigens. ELISA was chosen to screen and diagnose patients in residents of a low endemic area (Cristhiany et al., 2012; Turner et al., 2004). It was reported that the use of adult worm antigens (AWA) ELISA for detection of antibodies presents low specificity and cross interaction with other helminthes as hookworms. Also, the use of soluble egg antigen (SEA) ELISA has been shown to cross-react with Fasciola and it needs to be harvested from infected laboratory animals (Abdel-Fattah et al., 2011; Chand et al., 2010).

Schistosoma cercarial antigens (SCA) are easier than eggs and adult worm to produce and tests based on it are easier to scale up. Also, cercarial antibodies have been found to be at higher titers in early infection and produce far fewer false-positive results as it lacks epitopes responsible 
for cross reaction with other parasites (Knudsen et al., 2005; Sarhan et al., 2014).

Ionizing radiation was found to have various effects on helminthic parasites. Depending on the dose irradiated, parasites may be attenuated or died. It was reported that attenuation modifies expression of surface antigens and results in greater exposure of them converting the parasite into highly immunogenic however no biochemical or morphological changes can be demonstrated (Wuhrer et al., 1999). Radiation attenuated Schistosoma mansoni infective stage can be applied for control measures by immunoprotection or immunodiagnosis. Previous studies reported that vaccination with irradiation stimulates host immune response against challenge infection (Hewitson et al., 2005).

This study aims to comparatively evaluate normal and gamma-irradiated $S$. mansoni cercarial antigen by ELISA for serodiagnoses of chronic Schistosoma mansoni infected patients.

\section{MATERIAL AND METHODS}

\section{Sample collection and sera preparation}

Blood samples were collected from 52 iindividuals and classified into three groups. Group I included 21 patients who were positive for schistosomiasis from outpatient clinics of Menoufia University Hospitals and Mansoura University Hospitals, Egypt and were negative for other parasitic infections confirmed by examining stool samples by Kato-Katz technique. Group II included 18 patients who were negative for schistosomiasis and positive for other parasitic infections; (3) fascioliasis, (5) amoebiasis, (7) toxoplasmosis and (3) hydatidosis confirmed by parasitological and serological diagnosis. Group III included 13 healthy individuals (negative control). From each individual enrolled in this work, 5 $\mathrm{ml}$ blood was taken, centrifuged at $1000 \mathrm{xg}$ for $5 \mathrm{~min}$. After that, the serum was withdrawn, aliquted in $0.5 \mathrm{ml}$ eppendorf tubes and stored at $-80^{\circ} \mathrm{C}$ until used.

\section{Ethical approval and/or informed consent}

An informed consent was taken from all the patients after explaining the aim of the study to them the study protocol proved by research ethics committee of National Center for radiation Research and Technology (RECNCRRT).

\section{Preparation of the cercarial antigens}

$S$. mansoni cercariae were purchased from Schistosome Biological Supply Centre (SBSC), Theodor Bilharz Research Institute (TBRI), Cairo, Egypt. Half them were irradiated by exposure to 400 gray gamma irradiation at a dose rate $2.5 \mathrm{~K} . \mathrm{Gy} / \mathrm{h}$ at intensity of $400 \mu \mathrm{w} / \mathrm{cm}^{2}$ for one minute at the National Center for Radiation, Research and Technology (NCRRT) in Cairo, Egypt (Moawad et al., 2016). Antigens preparation were performed and stored at -70 until used according to the method of Feldmeier and Butter (1983).

\section{Enzyme linked immunosorbant assay (ELISA)}

Indirect ELISA was done as described by Santiago and Hillyer (1988) with some modifications. The optimal reaction conditions regarding antigens concentration, antibody and conjugate dilutions were chosen after preliminary checker-board titrations. For each cercarial antigen, the ELISA plates (Nunc, Denmark) were coated with $5 \mu \mathrm{g}$ dissolved in $100 \mu$ l coating buffer in each well. The plates were incubated overnight at $4^{\circ} \mathrm{C}$. In the next morning, the plates were washed with PBS/0.05\% Tween (T) to get rid of excess unbound antigen. The remaining free binding sites were blocked with 1\% BSA (bovine serum albumin) $(200 \mu \mathrm{l} / \mathrm{well})$, and kept for $1 \mathrm{~h}$ at room temperature. The plates were then washed 3 times with PBS/ $0.05 \% \mathrm{~T}$, the human sera were added to the plates in a final volume of $0.1 \mathrm{ml}$ diluted $1 / 100$ in PBS per well and the plates were incubated for 90 minutes at $37^{\circ} \mathrm{C}$. After that, the plates were washed with PBS/T, $0.1 \mathrm{ml} /$ well of conjugated horseradish peroxidase anti-human IgG (diluted at 1/1000) was added to all wells and incubated at $37^{\circ} \mathrm{C}$ for $1 \mathrm{~h}$. Finally, the plates were washed, $0.1 \mathrm{ml}$ ortho-phenylene-diamine substrate was added and the reaction was stopped by adding $100 \mu \mathrm{l} /$ well of $1 \mathrm{~N} \mathrm{NaOH}$. The reaction was read using ELISA reader (ELx800 Absorbance Reader Bio-Tek) at $405 \mathrm{~nm}$ wavelength. All OD readings above cut off value were considered positive. Cut off $=$ mean values of negative samples +2 SD according to Gool et al. (2002).

Statistical analysis was performed using one way ANOVA using statically package for social science version 15 (SPSS) computer programs (2002). Descriptive statistics as Mean and standard deviation (SD) were used as they measure central tendency and dispersion of quantitative data. Analytical statistics as comparing groups was done using Student's " $t$ " test for quantitative data of 2 independent samples and ANOVA (Analysis of Variance) for comparison of quantitative data of more than 2 groups. The following were used to calculate the corresponding dignostic parameters:

Sensitivity $=(a \div(a+c))^{*} 100$, Specificity $=(d \div(b+d))$ $* 100$, Positive predictive value $(\mathrm{PPV})=(\mathrm{a} \div(\mathrm{a}+\mathrm{d})) *$ 100 , Negative predictive value $(\mathrm{NPV})=(\mathrm{d} \div(\mathrm{c}+\mathrm{d})) * 100$, Diagnostic accuracy $=(\mathrm{a}+\mathrm{d}) \div(\mathrm{a}+\mathrm{b}+\mathrm{c}+\mathrm{d}) * 100$, Where $(\mathrm{a})$ is true positive, (b) is false positive, (c) is false negative, (d) is true negative. 


\section{RESULTS}

In the present work, total IgG, ELISA was adopted to compare the potency of the normal and irradiated cercarial antigens using $S$. mansoni and other parasitic infected sera. Reaction of $S$. mansoni infected sera towards normal and irradiated cercarial antigens revealed that there were $14 \mathrm{~S}$. mansoni infected sera showed higher antibodies response against irradiated SCA. The difference is highly significant when $\mathrm{P}<0.05$ (Fig. 1).

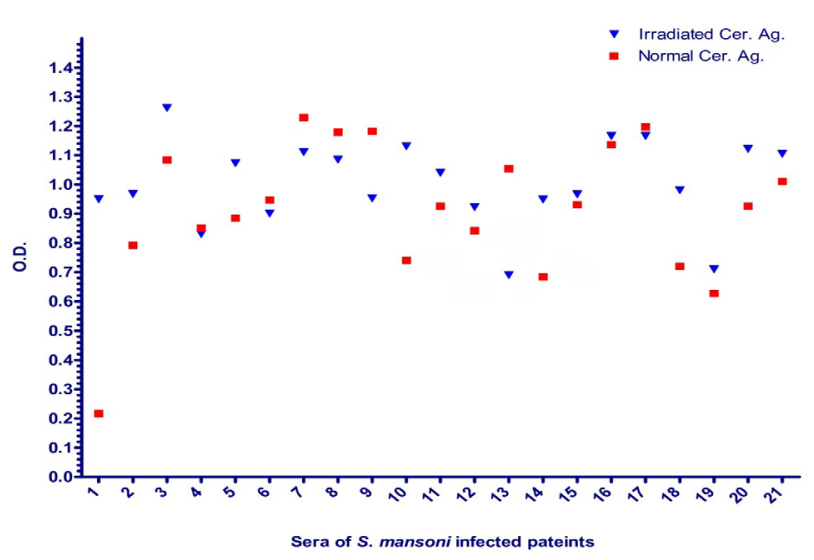

Fig. 1. Reactivity of the individual S. mansoni infected sera against irradiated SCA and normal SCA by ELISA.

Concerning cross reaction studies of the selected antigens, it was detected in Table I showing that normal SCA cross-reacted with 5 sera infected by other parasites which were 2 fascioliasis, 2 amoebiasis and 1 toxoplasmosis. While irradiated SCA cross-reacted with 4 sera infected by other parasites which were 1 fascioliasis and 3 amoebiasis.

Table I. Serological reactivity against normal and irradiated cercarial antigens using total IgG ELISA with other parasits infected serum.

\begin{tabular}{lll}
\hline Groups & \multicolumn{2}{l}{ No of sera $(+/-)$} \\
\cline { 2 - 3 } & Normal cercaria & Irradiated cercaria \\
\hline Group I (14) & $(21 / 21)$ & $(21 / 21)$ \\
Group II (18) & & \\
Fascioliasis (3) & $(2 / 3)$ & $(1 / 3)$ \\
Amoebiasis (5) & $(2 / 5)$ & $(3 / 5)$ \\
Toxoplasmosis (7) & $(1 / 7)$ & $(0 / 7)$ \\
Hydatidosis (3) & $(0 / 5)$ & $(0 / 3)$ \\
Group III (13) & $(0 / 13)$ & $(0 / 13)$ \\
\hline
\end{tabular}

The cutoff value for ELISA test using normal and irradiated cercarial antigen with sera were evaluated as 0.18 and 0.168 . Optical density values of the normal and irradiated SCA against schistosomasis sera were ranged from 0.217-1.229 and 0.690-1.262. Optical density values of the positively cross reacted normal SCA with amoebiasis, fascioliasis and toxoplasmosis ranged from $0.55-1.89,0.19-0.95$ and 0.95 respectively. While irradiated SCA cross reacted with amoebiasis OD ranged from $0.22-1.85$ and with fascioliasis at 0.96 (Fig. 2).
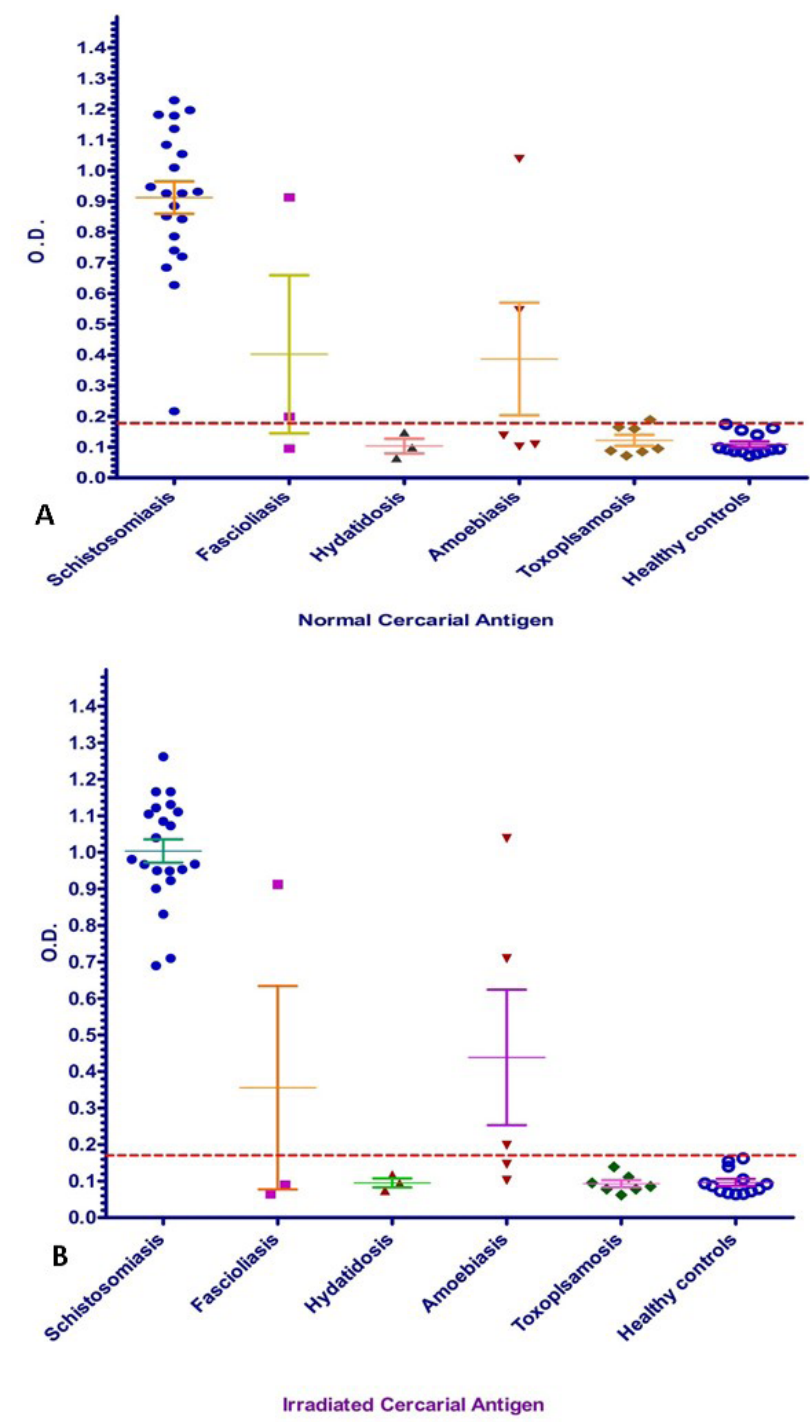

Fig. 2. Serodiagnosis of human schistosomiasis mansoni and other parasites using ELISA- normal (A) and irradiated (B) SCA Cut-off of healthy controls.

Both antigens revealed same sensitivity (100\%) and NPV (100\%). Irradiated SCA revealed higher specificity 
(87\%) compared to normal SCA (83.9). Higher PPV was obtained from Irradiated SCA (84\%) while normal SCA achieved $80.7 \%$. The accuracy of Irradiated SCA was 92.3 and normal SCA was $90.4 \%$ (Table II).

Table II. Sensitivity, Specificity, PPV, NPV and diagnostic accuracy percentages of both irradiated SCA and normal SCA by ELISA.

\begin{tabular}{llllll}
\hline $\begin{array}{l}\text { Accuracy } \\
\%\end{array}$ & $\begin{array}{l}\text { NPV } \\
\%\end{array}$ & $\begin{array}{l}\text { PPV } \\
\%\end{array}$ & $\begin{array}{l}\text { Specific- Sensitiv- Antigen } \\
\text { ity \% }\end{array}$ & $\begin{array}{l}\text { S } \% \\
\text { ity }\end{array}$ & \\
\hline $92.3 \%$ & $100 \%$ & $84 \%$ & $87 \%$ & $100 \%$ & Irradiated SCA \\
$90.4 \%$ & $100 \%$ & $80.7 \%$ & $83.9 \%$ & $100 \%$ & Normal SCA. \\
\hline
\end{tabular}

\section{DISCUSSION}

Although great efforts worldwide have been made to control schistosomiasis, it continues to spread to new geographic areas. The problem is linked with diagnosis as we still depend on microscopic examination which proved to be not ideal as light infections are often missed so there is a need to develop practical and scalable techniques (Coulibaly et al., 2016).

Serological examinations as screening for antischistosomal antibodies are of limited use for the diagnosis, as large parts of the population may carry antibodies due to past infections so there is an urge for finding the best antigen that can be used in ELISA to diagnose patients from endemic area and follow-up treatment (Kinkel et al., 2012).

In the current study, two types of cercarial antigens were enrolled normal and gamma-irradiated one. All the positive sera as judged by microscopic examination reacted positively with the two antigens. The cutoff value of the examined serum samples of Schistosomasis mansoni patient against normal cercariae antigen were 0.18 and 0.168 . These results nearly coincides with previous study revealed the cutoff value for ELISA test using Schistosoma adult worm, cercarial and egg antigens with sera of infected patients as $0.64,0.82$, and 0.892 respectively (El-Aswad et al., 2011).

Studying the antibodies response towards either antigen, it was found that there were 14 Schistosoma mansoni infected sera showed higher antibodies response against the irradiated SCA than that of the normal one. Both antigens achieved $100 \%$ sensitivity while irradiated SCA showed $87 \%$ specificity rather than normal one giving $83.9 \%$. In the same context, previous studies revealed 86.6 $\%$ sensitivity and $94.11 \%$ specificity on assessing total IgG ELISA SCA using sera of chronic schistosomiasis patients. Also, ELISA using cercarial transformation fluid showed sensitivity of $89.7 \%$ and an estimated specificity of $100 \%$ when used in non-endemic regions (El-Aswad et al., 2011; Eberl et al., 2001). While in endemic areas, the sensitivity has been reported to be between 20 and 65 $\%$ which could be explained that in endemic areas people may have subclinical infection with low parasite burden but have high antibodies titers (Doenhoff et al., 2004).

The current study clarified cross reactivity between schistosomiasis, fascioliasis, toxoplasmosis and amoebiasis that was detected using normal SCA while irradiated SCA only cross-reacted with fascioliasis and amoebiasis. This was in accordance to results exhibited by Abdel-Fattah et al. (2011) reported serological cross-reactivity of SEA and SCA with sera from Trichinella, Fasciola and Hydatid infections. This may be too high for mass population work causing poor specificity especially in areas where other helminthes are prevalent.

The findings of present study revealed that irradiated SCA achieved better PPV and accuracy ( $84 \%$ and 92.3) than t normal SCA achieving $80.7 \%$ and $90.4 \%$. These coincides with studies reported that radiation induces changes in the surface molecule making it highly immuonogic. When ceracriae exposed to attenuated dose of gamma irradiation, antigens present on the surface will be altered and certain cryptic antigens having large antigenic loads exposed. Also radiation affects some structural and motor proteins, enzymes in addition to carbohydrates on the parasite surface (Wuhrer et al., 1999; Yang et al., 2009).

It could be concluded that there was a difference between normal and gamma radiation attenuated Schistosoma mansoni cercarial antigens in diagnosis of chronic schistosomiasis using ELISA. Irradiated SCA achieved better remarkable ODs, specificity due to low cross-reactivity with other parasites and better diagnostic accuracy so it is an effective tool to make a correct diagnosis.

\section{ACKNOWLEDGEMENTS}

The authors wish to acknowledge the assistance given by the National Center of Radiation Research and Technology, which provided all facilities for this study.

\section{Statement of conflict of interest}

The authors have declared no conflict of interest.

\section{Funding}

This research did not receive any specific grant from funding agencies in the public, commercial, or not-forprofit sectors. 


\section{REFERENCES}

Abdel-Fattah, M.M., Al-Sherbiny, M., Osman, A., Sharmy, R. and Tsang, V., 2011. Improving the detection limit of quantitative diagnosis of anti-S. haematobium antibodies using Falcon Assay Screening Tet (FSAT) ELISA by developing a new standard curve. Parasitol. Res., 108: 1457-1463. https://doi.org/10.1007/s00436-010-2198-y

Amin, M.A., Elsadig, A.M. and Osman, H.A., 2017. Evaluation of cathodic antigen urine tests for diagnosis of Schistosoma mansoni infection in Sudan. Saudi J. med. Sci., 5: 56-61. https://doi. org/10.4103/1658-631X.194257

Cavalcanti, M.G., Silva, L.F., Peralta, R.H., Barreto, M.G.M. and Peralta, J.M., 2013. Schistosomiasis in areas of low endemicity: a new era in diagnosis. Trends Parasitol., 29: 75-82. https://doi. org/10.1016/j.pt.2012.11.003

Chand, M.A., Chiodini, P.L. and Doenhoff, M.J., 2010. Development of a new assay for the diagnosis of schistosomiasis, using cercarial antigens. Trans. R. Soc. Trop. Med. Hyg., 104: 255-258. https://doi. org/10.1016/j.trstmh.2009.12.004

Coulibaly, J.T., Ouattara, M., Becker, S.L., Lo, N.C., Keiser, J., N'Goran, E.K., Ianniello, D., Rinaldi, L., Cringoli, G. and Utzinger, J., 2016. Comparison of sensitivity and faecal egg counts of Mini-FLOTAC using fixed stool samples and Kato-Katz technique for the diagnosis of Schistosoma mansoni and soiltransmitted helminths. Acta Trop., 164: 107-116. https://doi.org/10.1016/j.actatropica.2016.08.024

Cristhiany, M., Pinheirol, C., Carneiro, T.R., Hanemann, A., de Oliveira, S.M., Schemelzer, F. and Bezerra, M., 2012. The combination of three faecal parasitological methods to improve the diagnosis of schistosomiasis mansoni in a low endemic setting in the state of Ceará Brazil. Mem. Inst. Oswaldo Cruz, Rio de Janeiro, 107: 873-876. https://doi. org/10.1590/S0074-02762012000700006

Doenhoff, M.J., Chiodini, J.P.L. and Hamilton, J.V., 2004. Specific and sensitive diagnosis of Schistosoma infection: can it be done with antibodies? Trends Parasitol., 20: 35-39. https:// doi.org/10.1016/j.pt.2003.10.019

Eberl, M., Langermans, J.A., Vervenne, R.A., Nyame, A.K., Cummings, R.D., Thomas, A.W., Coulson, P.S. and Wilson, A., 2001. Antibodies to glycans dominate the host response to schistosome larvae and eggs: Is their role protective or subversive? J. Infect. Dis., 183: 1238-1247. https://doi. org/10.1086/319691

El-Aswad, B.W., Doenhoff, M.J., El Hadidi, A.S., Schwaeblea, W.J. and Nicholas, J.L., 2011. Use of recombinant calreticulin and cercarial transformation fluid (CTF) in the serodiagnosis of Schistosoma mansoni. Immunobiology., 216: 379385. https://doi.org/10.1016/j.imbio.2010.06.014

Feldmeier, H. and Butter, D.W., 1983. Immunodiagnosis of schistosomiasis hematobium and schistosomiasis mansoni in man. Application of crude extract from adult worms and cercariae in the IHA and ELISA. Zentralbl. Bakteriol. Mikrobiol. Hyg., $A$, 255: 413-421. https://doi.org/10.1016/S01743031(83)80185-1

Gool, T. V., Vetter, H., Vervoort, T., Doenhoff, M. J., Wetsteyn, J. and Overbosch, D., 2002. Serodiagnosis of imported schistosomiasis by a combination of a commercial indirect hemagglutination test with Schistosoma mansoni adult worm antigens and an enzyme-linked immunosorbent assay with $S$. mansoni egg antigens. J. clin. Microbiol., 40: 34323437. https://doi.org/10.1128/JCM.40.9.34323437.2002

He, P., Gordon, C.A., Williams, G.M., Li, Y., Wang, Y., Hu, J., Gray, D.J., Ross, A.G., Harn, D. and Mcmanus, D.P., 2018. Real-time PCR diagnosis of Schistosoma japonicum in low transmission areas of China. Infect. Dis. Poverty, 7: 1-11. https:// doi.org/10.1186/s40249-018-0390-y

Hewitson, J.P., Hamblin, P.A. and Mountford, A.P., 2005. Immunity induced by the radiationattenuated schistosome vaccine. Parasite Immunol., 27: 271-280. https://doi.org/10.1111/j.13653024.2005.00764.x

Kinkel, H.F., Dittrich, S., Baumer, B. and Weitzela, T., 2012. Evaluation of eight serological tests for diagnosis of imported schistosomiasis. Clin. Vaccine Immunol., 19: 948-953. https://doi. org/10.1128/CVI.05680-11

Knudsen, G.M., Medzihradszky, K.F., Lim, K.C., Hansell, E. and McKerrow, J.H., 2005. Proteomic analysis of Schistosoma mansoni cercarial secretions. Mol. Cell Proteomics, 4: 18621875. https://doi.org/10.1074/mcp.M500097MCP200

Ludolf, F., Patrocinio, P.R., Corre^a-Oliveria, R., Gazzinelli, A., Falcone, F.H., Teixeira-Ferreira, A., Perales, J., Oliveira, G.C. and Silva-Pereira, R.A., 2014. Serological screening of the Schistosoma mansoni adult worm proteome. PLoS. Negl. Trop. Dis., 8: e2745. https://doi.org/10.1371/journal. pntd.0002745 
Moawad, M.A, Amin, M.M. and Hafez, E.N., 2016. Hepatic histopathological and histochemical changes in mice infected with Schistosoma Mansoni after vaccination with gamma radiation attenuated schistosomules. Arab J. Nucl. Sci. Appl., 94: 188-198.

Santiago, N. and Hillyer, G.V., 1988. Antibody profiles by EITB and ELISA of cattle and sheep infected with Fasciola hepatica. J. Parasitol., 74: 810 - 818. https://doi.org/10.2307/3282259

Sarhan, R.M., Aminou, H.A., Saad, G.A.R. and Ossama, A.A., 2014. Comparative analysis of the diagnostic performance of adult, cercarial and egg antigens assessed by ELISA, in the diagnosis of chronic human Schistosoma mansoni infection. Parasitol. Res., 113: 3467-3476. https://doi.org/10.1007/ s00436-014-4017-3

Siqueira, L.M.V., Coelho, P.M.Z., de Oliveira, A.A., Massara, C.L., Carneiro, N.F.F., Lima, A.C.L. and Enk, M.J., 2011. Evaluation of two coproscopic techniques for the diagnosis of schistosomiasis in a low transmission area in the state of Minas
Gerais, Brazil. Mem. Inst. Oswaldo. Cruz., 106: 844-850. https://doi.org/10.1590/S007402762011000700010

Turner, P., Lalloo, K., Bligh, J., Armstrong, M., Whitty, C.J.M., Doenhoff, M.J. and Chiodmi, P.L., 2004. Serological specification of human schistosome infections by ELISA with a panel of three antigens. J. clin. Pathol., 57: 1193-1196. https://doi. org/10.1136/jcp.2003.014779

Wuhrer, M., Dennis, R.D., Doenhoff, M.J., Bickle, Q., Lochnit, G. and Geyer, R., 1999. Immunochemical characterization of Schistosoma mansoni glycolipid antigens. Mol. Biochem. Parasitol., 155: 155-169. https://doi.org/10.1016/S0166-6851(99)00123-1

Yang, L.L., Lv, Z.Y., Hu, S.M., He, S.J., Li, Z.Y., Zhang, S.M., Zeng, H., LI, m., Yu, X., Fung, M.C. and Wu, Z., 2009. Schistosoma japonicum: proteomics analysis of differentially expressed proteins from ultraviolet-attenuated cercariae compared to normal cercariae. Parasitol. Res., 105: 237-248. https://doi.org/10.1007/s00436-009-1387-z 